HNO 2022 $\cdot 70: 520-532$

https://doi.org/10.1007/s00106-021-01139-5

Angenommen: 22. November 2021

Online publiziert: 21. Januar 2022

๑ ( Der/die Autor(en) 2022

\section{Wirksamkeit der \\ Hörgeräteversorgung bei hochgradigem Hörverlust}

Max Engler · Frank Digeser · Ulrich Hoppe

Audiologische Abteilung, Hals-Nasen-Ohrenklinik, Kopf- und Halschirurgie, Universitätsklinikum Erlangen, Erlangen, Deutschland

\title{
Zusammenfassung
}

Hintergrund: In der Praxis liegt das unilaterale Einsilberverstehen mit Hörgerät bei $65 \mathrm{~dB}$ SPL ( $\mathrm{EV}_{65}(\mathrm{HG})$ ) häufig unter dem maximalen Einsilberverstehen aus dem Sprachaudiogramm ( $\mathrm{mEV}$ ), insbesondere bei Hörgeräteträgern mit hochgradigem Hörverlust. Diese Arbeit zielte darauf ab, den Wirkungsgrad Q der Hörgeräteversorgung, den Quotienten aus $\mathrm{EV}_{65}(\mathrm{HG})$ und $\mathrm{mEV}$, bei Hörgeräteträgern mit hochgradigem bis an Taubheit grenzendem Hörverlust zu untersuchen.

Material und Methoden: Es wurden Daten aus In-situ-Messungen, dem Reinton- und Sprachaudiogramm und dem Sprachverstehen mit und ohne Hörgerät von 93 Ohren von 64 Patienten ausgewertet. Die Patienten stellten sich im Jahr 2019 für eine Hörgerätekontrolle in unserem Hörzentrum vor. Es wurden die Abweichung der insitu gemessenen frequenzabhängigen Ausgangspegelwerte von den Zielvorgaben der präskriptiven Anpassformeln NAL-NL2 und DSL v5.0 analysiert. Für die Parameter $\mathrm{EV}_{65}(\mathrm{HG})$ und $\mathrm{Q}$ wurden jeweils die Spearman-Korrelationskoeffizienten für den Sprachverständlichkeitsindex (SII) berechnet.

Ergebnisse: Bei mehr als $67 \%$ der Hörgeräteeinstellungen stimmten die Ausgangspegelwerte mit den Zielkurven für NAL-NL2 oder DSL v5.0 im Bereich von $\pm 5 \mathrm{~dB}$ für Frequenzen von 0,5 bis $4 \mathrm{kHz}$ für $65 \mathrm{~dB}$ SPL überein. Trotzdem wurde das $\mathrm{mEV}$ mit Hörgerät bei $65 \mathrm{~dB}$ SPL nicht erreicht (mittlere Abweichungen: 34,4\%). EV $65(\mathrm{HG})$ und Q waren jedoch am besten, wenn Zielwerte für DSL v5.0 bei $65 \mathrm{~dB}$ SPL erreicht wurden, was mit einem höheren SIl einhergeht.

Schlussfolgerung: Für Hörgeräteträger mit hochgradigem bis an Taubheit grenzendem Hörverlust führen die Anpassformeln NAL-NL2 und DSL v5.0 nicht zu einer solchen Verstärkung, dass bei Alltagssprache von $65 \mathrm{~dB}$ SPL das mEV erreicht wird. Es bleibt zu untersuchen, ob alternative Präskriptionen mit besserer Hörbarkeit für Eingangspegel von 50 und 65 dB SPL den Wirkungsgrad der Hörgeräteversorgung verbessern könnten.

\section{Schlüsselwörter}

Freiburger Sprachtest · Maximales Einsilberverstehen · Sprachaudiometrie · In-situ-Messung • Hochgradiger bis an Taubheit grenzender Hörverlust

\section{Hintergrund}

Die Prävalenz von Hörstörungen liegt weltweit bei 20,4\%, wobei die Mehrheit der Menschen nur von einem geringgradigem Hörverlust betroffen ist [25]. Der Anteil der Menschen mit hochgradigem bis an Taubheit grenzendem Hörverlust beträgt 0,8\% an der Gesamtbevölkerung bzw. 4\% an den Menschen mit Hörbeeinträchtigung [25]. Der mittlere Hörverlust, der Mittelwert aus den vier Luftleitungshörschwel- len bei 0,$5 ; 1 ; 2$ und $4 \mathrm{kHz}$ (,four-frequency pure-tone average", 4FPTA), liegt in dieser Gruppe bei $\geq 65 \mathrm{~dB}$ HL. Für die Prävalenz von Hörstörungen in Deutschland finden sich in der Literatur ähnliche Werte zwischen 16 und $25 \%$, jeweils abhängig von Alter, Studienumgebung, Definition der Hörminderung und Messmethodik [15, 22, 23]. Da für die meisten Hörstörungen keine kausalen Therapien zur Verfügung stehen, werden in der Regel Hörgeräteversorgungen durchgeführt, um das Sprachverste- 
hen in Ruhe und im Störgeräusch zu verbessern. Insbesondere bei hochgradigem Hörverlust ist die Hörgeräteanpassung eine Herausforderung für den Hörakustiker.

Aus dem Reintonaudiogramm allein lassen sich keine hinreichenden Rückschlüsse auf das mit Hörgerät erreichbare Sprachverstehen ziehen $[5,9,17]$. Um den Versorgungserfolg bewerten zu können, werden als Parameter das über Kopfhörer ermittelte maximale Einsilberverstehen (mEV) aus dem Sprachaudiogramm und das Einsilberverstehen im Freifeld bei einem Schalldruckpegel von $65 \mathrm{~dB}$ SPL mit Hörgerät $\left(\mathrm{EV}_{65}(\mathrm{HG})\right)$ und ohne Hörgerät $\left(E_{65}\right)$ hinzugezogen. In der HilfsmittelRichtlinie (HilfsM-RL) wird ein Hörgewinn von mindestens $20 \%$-Punkten gegenüber der unversorgten Situation bei $65 \mathrm{~dB}$ SPL gefordert [6]. Zusätzlich soll das $\mathrm{EV}_{65}(\mathrm{HG})$ dem mEV "möglichst nahe" kommen [6]. Untersuchungen von älteren Hörgeräteträgern über 60 Jahre zeigten zwar einen Gewinn durch die Hörgeräteversorgung in $82 \%$ der Fälle, welcher allerdings in
$56 \%$ der Fälle unter den 20\%-Punkten lag [14]. Nach Schorn et al. [19, 20] und Kießling et al. [13] gilt eine Hörgeräteversorgung als angemessen, sofern die Abweichungen höchstens 5\%-Punkte [19, 20] bis $10 \%$-Punkte [13] betragen. Andere Studien zeigten jedoch, dass das mittlere unilaterale $\mathrm{EV}_{65}(\mathrm{HG})$ ca. $20 \%$-Punkte unter dem $m E V$ liegt $[5,9,17]$. Die in der Praxis geforderte Annäherung des $\mathrm{EV}_{65}(\mathrm{HG})$ an das $m E V$ wird in der unilateralen Situation von 77 [9] bis $81 \%$ [14] der Probanden nicht erreicht. Als Erklärung hierfür wurde bereits der geringe Abstand des Pegels, an dem das maximale Einsilberverstehen erreicht wird $\left(L_{m E v}\right)$, von der Unbehaglichkeitsschwelle genannt, welcher bei $55 \%$ der untersuchten Ohren unter $10 \mathrm{~dB}$ lag [9]. Diese geringe Dynamikreserve führt dazu, dass eine adäquate Verstärkungseinstellung, welche nötig wäre, um zu einem angemessenen Sprachverstehen zu gelangen, nahe an die Unbehaglichkeitsschwelle heranführen würde. Beide Parameter $\mathrm{mEV}$ und $\mathrm{EV}_{65}(\mathrm{HG})$ nehmen

Abkürzungen

\begin{tabular}{|c|c|}
\hline$d B$ & Dezibel \\
\hline$D S L v 5.0$ & Desired Sensation Level version 5.0 \\
\hline$S L_{ \pm 5}$ & $\begin{array}{l}\text { Gruppierung von Ohren: mittleres LTASS bei } 65 \mathrm{~dB} \text { SPL mit maximalen Abweichungen von } \\
\pm 5 \mathrm{~dB} \text { von DSL-v5.0-Zielkurve }\end{array}$ \\
\hline$S L>5$ & Gruppierung von Ohren: mittleres LTASS bei $65 \mathrm{~dB}$ SPL > $5 \mathrm{~dB}$ über DSL-v5.0-Zielkurve \\
\hline$E V_{65}$ & Einsilberverstehen in Ruhe bei $65 \mathrm{~dB}$ SPL im Freifeld \\
\hline$V_{65}(H G)$ & Einsilberverstehen in Ruhe bei $65 \mathrm{~dB}$ SPL im Freifeld mit Hörgerät \\
\hline IS & Luftleitungshörschwelle \\
\hline ISTS & Internationales Sprach-Test-Signal („,international speech test signal“) \\
\hline$L_{E-50,-65,-80}$ & Eingangspegel von 50,65 und $80 \mathrm{~dB}$ SPL \\
\hline$L_{m E V}$ & Pegel beim maximalen Verstehen im Freiburger Einsilbertest \\
\hline LTASS & Mittleres Langzeit-Sprachspektrum („long-term average speech spectrum“) \\
\hline$m E V$ & Maximales Verstehen im Freiburger Einsilbertest \\
\hline$N A L-N L 2$ & National Acoustic Laboratories-Nonlinear version 2 \\
\hline$N A L_{<5}$ & Gruppierung von Ohren: mittleres LTASS bei $65 \mathrm{~dB}$ SPL $<5 \mathrm{~dB}$ unter NAL-NL2-Zielkurve \\
\hline$N A L_{ \pm 5}$ & $\begin{array}{l}\text { Gruppierung von Ohren: mittleres LTASS bei } 65 \mathrm{~dB} \text { SPL mit maximalen Abweichungen von } \\
\pm 5 \mathrm{~dB} \text { von NAL-NL2-Zielkurve }\end{array}$ \\
\hline Q & Wirkungsgrad der Hörgeräteversorgung \\
\hline$R E A R$ & Ausgangsschalldruckpegel des Hörgeräts vor dem Trommelfell („real-ear aided response”) \\
\hline$R E C D$ & $\begin{array}{l}\text { Differenz zwischen dem Schalldruckpegel im Ohr und dem Schalldruckpegel im Kuppler } \\
\text { („,real-ear to coupler difference“) }\end{array}$ \\
\hline REIG & Verstärkung durch das Hörgerät, REIG = REAR-REUR („real-ear insertion gain“) \\
\hline REM & Echt-Ohr-Messungen („real-ear Measurements“) \\
\hline RETSPL & $\begin{array}{l}\text { Äquivalenter Bezugs-Schwellenschalldruckpegel („reference equivalent threshold sound } \\
\text { pressure level“) }\end{array}$ \\
\hline REUR & Schalldruckpegel im unversorgten Ohr vor dem Trommelfell („real-ear unaided response”) \\
\hline SII & Sprachverständlichkeitsindex („,speech intelligibility index“) \\
\hline SPL & Schalldruckpegel („sound pressure level“) \\
\hline US & Unbehaglichkeitsschwelle \\
\hline 4FPTA & $\begin{array}{l}\text { Mittelwert aus den vier Luftleitungshörschwellen bei } 0,5 ; 1 ; 2 \text { und } 4 \mathrm{kHz} \text { (,four-frequency } \\
\text { pure-tone average") }\end{array}$ \\
\hline$\triangle N A L / \triangle D S L$ & $\begin{array}{l}\text { Differenz zwischen mittlerem LTASS und mittlerer Zielkurve für NAL-NL2 und DSL v5.0 im } \\
\text { Frequenzbereich von } 0,5-4 \mathrm{kHz}\end{array}$ \\
\hline
\end{tabular}

mit zunehmendem Hörverlust ab, und bei einem 4FPTA $\geq 60 \mathrm{~dB}$ HL zeigt sich zusätzlich eine große interindividuelle Varianz [9]. Der $L_{m E v}$ nimmt mit zunehmendem Hörverlust zu, sodass das mEV nicht nur kleiner ausfällt, sondern auch erst bei höheren Pegeln erreicht wird [9].

Um die Verstärkung von Hörgeräten beurteilen zu können, werden In-situMessungen ohne (,real-ear unaided response“, REUR) und mit Hörgerät (,realear aided response", REAR) durchgeführt. Mit der REUR-Messung wird die Übertragungsfunktion des offenen Gehörgangs ermittelt, indem der Schallpegel am Trommelfell mit einem Sondenmikrofon gemessen wird. Für die Messung der REAR wird der Schallpegel am Trommelfell mit dem platzierten und eingeschalteten Hörgerät gemessen. Die Differenz von REAR und REUR wird als wirksame akustische Verstärkung („real-ear insertion gain“, REIG) bezeichnet. Als Testsignal wird das universelle internationale Sprach-Test-Signal (,international speech test signal“, ISTS [8]) verwendet. Mit dieser Vorgehensweise wird der physikalische Einfluss der Anatomie des Außenohrs (Ohrmuschel und Gehörgangsresonanz), der Ankopplung des Hörgeräts an das Ohr (Otoplastik oder Dome) sowie der Art des Schallwandlers (Schallschlauch, externer Hörer oder In-dem-Ohr-Gerät) auf den Pegel des Ausgangssignals mitberücksichtigt. Die gemessene REAR kann anschließend mit theoretischen Zielvorgaben verglichen werden. Die bedeutendsten herstellerunabhängigen Vorschriften zur Bestimmung der Zielwerte sind die beiden präskriptiven Anpassformeln NAL-NL2 („National Acoustic Laboratories-Nonlinear version 2", [12]) und DSL v5.0 („Desired Sensation Level version 5.0", [21]). Mit diesen Anpassformeln lassen sich frequenzabhängige Zielkurven für geringe ( $50 \mathrm{~dB} \mathrm{SPL})$, mittlere $(65 \mathrm{~dB} \mathrm{SPL})$ und hohe $(80 \mathrm{~dB} \mathrm{SPL})$ Eingangspegel berechnen.

\section{NAL-NL2}

NAL-NL2 wurde mit dem Ziel entwickelt, das Sprachverstehen zu maximieren und dabei die Lautheit auf diejenige von normalhörenden Menschen zu begrenzen [12]. Dafür wurde ein Sprachverständlichkeitsmodell, welches auf dem Sprachver- 
ständlichkeitsindex (,sspeech intelligibility index", SII [1]) basiert, sowie ein Lautheitsmodell nach Moore \& Glasberg [16] in einem adaptiven Optimierungsprozess verwendet. Zunächst wird die frequenzabhängige Verstärkung bestimmt, um das Sprachverstehen zu maximieren. Anschließend wird die empfundene Lautheit abgeschätzt und mit der Lautheit verglichen, die ein Mensch ohne Hörbeeinträchtigung in derselben Situation empfinden würde. Liegt die empfundene Lautheit des Hörbeeinträchtigten über der von normalhörenden Menschen, wird die Gesamtverstärkung reduziert. Neben einer binauralen Korrektur berücksichtigt NAL-NL2 unter anderem auch einen möglichen Schallleitungsanteil, die Anzahl der Frequenzkanäle, die Hörgeräteerfahrung und die Ankopplung des Hörgeräts an das Ohr (Otoplastik und Durchmesser der Zusatzbohrung oder Dome).

\section{DSL v5.0}

Das Ziel von DSL v5.0 ist es, möglichst viele für das Sprachverstehen relevante Sprachanteile in den hörbaren Bereich innerhalb der Restdynamik zu bringen, ohne dabei die Unbehaglichkeit zu erreichen (Lautheitsnormalisierung) [3, 21]. Im Gegensatz zu NAL-NL2 wird außerdem die gemessene Unbehaglichkeitsschwelle berücksichtigt.

Der wesentliche Unterschied zwischen den resultierenden Zielkurven der beiden Anpassformeln ist, dass mit steigendem Hörverlust bei DSL v5.0 typischerweise mehr Verstärkung für mittlere und hohe Eingangspegel berechnet wird als bei NALNL2 [4, 18].

Im Rahmen der routinemäßig in unserem Hörzentrum durchgeführten Hörgeräteüberprüfungen werden neben den üblichen Sprachverständlichkeitsmessungen auch die oben beschriebenen objektiven In-situ-Messungen durchgeführt. Da die Hörgeräteanpassungen extern von Hörakustikern außerhalb der Klinik durchgeführt werden, ist nicht bekannt, nach welchen Kriterien die Anpassung der Hörgeräte erfolgte. Die standardisierte Vorgehensweise in unserem Hause besteht daher aus dem Vergleich der in-situ gemessenen REAR sowohl mit den Zielkur- ven gemäß NAL-NL2 als auch mit denen gemäß DSL v5.0.

Kürzlich wurde von Dörfler et al. [5] ein Schätzwert für den sprachaudiometrischen Wirkungsgrad Q der Hörgeräteversorgung eingeführt. Dieser berechnet sich als Quotient aus $\mathrm{EV}_{65}(\mathrm{HG})$ und $\mathrm{mEV}$ $\left(\mathrm{Q}=\mathrm{EV}_{65}(\mathrm{HG}) / \mathrm{mEV}\right)$ und beschreibt, welcher Anteil des mEV mit dem Hörgerät bei 65 dB SPL umgesetzt werden kann. Dörfler et al. berichteten, dass der Wirkungsgrad in vielen Fällen deutlich unter 1 liegt und mit steigendem Hörverlust abfällt.

Ziel der vorliegenden Studie war es, bei Hörgeräteträgern mit hochgradigem bis an Taubheit grenzendem Hörverlust Zusammenhänge zwischen der gemessenen REAR und dem Sprachverstehen mit Hörgerät bzw. dem Wirkungsgrad Q zu beschreiben. Hierzu wurden die Ohren hinsichtlich der Abweichungen der REAR von den Zielkurven gruppiert und für diese der SIl mit dem Sprachverstehen mit Hörgerät bzw. dem Wirkungsgrad korreliert.

\section{Patienten und Methoden}

\section{Patienten}

Für diese retrospektive Studie wurden 100 klinisch durchgeführte Routinekontrollen von Hörgeräteträgern analysiert, wobei insgesamt Messungen von 152 Ohren betrachtet wurden. Die Kontrollen fanden in der Zeit von 08/2019 bis 12/2020 in der Erlanger HNO-Klinik statt. Eingeschlossen wurden uni- und bilateral versorgte Hörgeräteträger sowie bimodal versorgte Patienten, welche auf dem Hörgeräteohr einen 4FPTA von $60 \mathrm{~dB}$ HL oder mehr aufwiesen. Weitere Einschlusskriterien waren Deutsch als Muttersprache, vollendetes 18. Lebensjahr, eine abgeschlossene technische Anpassung der Hörgeräte beim jeweiligen Hörakustiker und mindestens 3 Monate Hörgeräteerfahrung. Die letzte Überprüfung und Nachjustierung der Hörgeräteeinstellung lag maximal 6 Monate zurück. Die Ausschlusskriterien waren eine mittlere Differenz von Luft- und Knochenleitungsschwellen zwischen 0,5 und $4 \mathrm{kHz}$ von mehr als $5 \mathrm{~dB}$ und erkennbare technische Defekte an den Hörgeräten. Es verblieb ein Kollektiv von 64 Patienten mit uni- oder bilateralem, hochgradigem oder an Taubheit grenzendem Hörverlust
(33 Frauen, 31 Männer) im Alter von 24-90 Jahren (Mittelwert und Standardabweichung: $65,8 \pm 14,8$ Jahre). Daraus resultierten 93 Ohren (48 links, 45 rechts) mit einem 4FPTA von 60,0-105,3 dB HL (Mittelwert und Standardabweichung: $72,9 \pm 11,6 \mathrm{~dB} \mathrm{HL})$.

\section{Ton- und Sprachaudiometrie}

Mittels Reintonaudiometrie wurden die Luftleitungsschwellen für Frequenzen zwischen 0,125 und $0,5 \mathrm{kHz}$ in Oktavschritten und anschließend bis $8 \mathrm{kHz}$ in Halboktavschritten bestimmt. Die Messung der Knochenleitungs- sowie Unbehaglichkeitsschwellen erfolgte für Frequenzen zwischen 0,5 und $4 \mathrm{kHz}$ in Oktavschritten. Die Messungen des Sprachverstehens wurden in Ruhe und in unilateraler Darbietung mit dem Freiburger Einsilbertest [7] durchgeführt. Es erfolgte eine randomisierte Auswahl der Freiburger Listen, wobei die Listen 1, 3, 5, 14 und 15 nicht verwendet wurden [9]. Zunächst wurde das Sprachverstehen bei $65 \mathrm{~dB}$ SPL über Kopfhörer ermittelt. Daraufhin wurde der Präsentationspegel in geeigneter Schrittweite (üblicherweise $10 \mathrm{~dB}$, bei geringer Restdynamik $5 \mathrm{~dB}$ ) solange erhöht, bis ein Einsilberverstehen von $100 \%$ oder der kleinste nicht mehr tolerierbare Pegel (Unbehaglichkeitsschwelle für Sprache) bzw. die Audiometerleistungsgrenze erreicht wurde. Anschließend wurde im freien Schallfeld über Lautsprecher für jede Seite einzeln der Freiburger Einsilbertest in Ruhe ohne und mit Hörgerät durchgeführt. Die Gegenseite wurde dabei regelgerecht maskiert. Die Freifeldmessungen mit Hörgerät erfolgten in der Regel mit zwei Listen zu 20 Wörtern. Alle Messungen wurden mit einem Audiometer vom Typ AT1000 (Fa. Auritec GmbH, Hamburg, Deutschland) durchgeführt. Das maximale Einsilberverstehen (mEV), der Pegel, bei dem das mEV erreicht wird ( $\left.L_{m E V}\right)$ [10] sowie das Einsilberverstehen ohne $\left(E_{65}\right)$ und mit Hörgerät $\left(E_{65}(H G)\right)$ wurden dokumentiert.

\section{In-situ-Messungen}

Über In-situ-Messungen mit der Aurical II (Fa. NATUS GmbH \& Co. KG, Trier, Deutschland) wurde das Hörgerät direkt am Patien- 


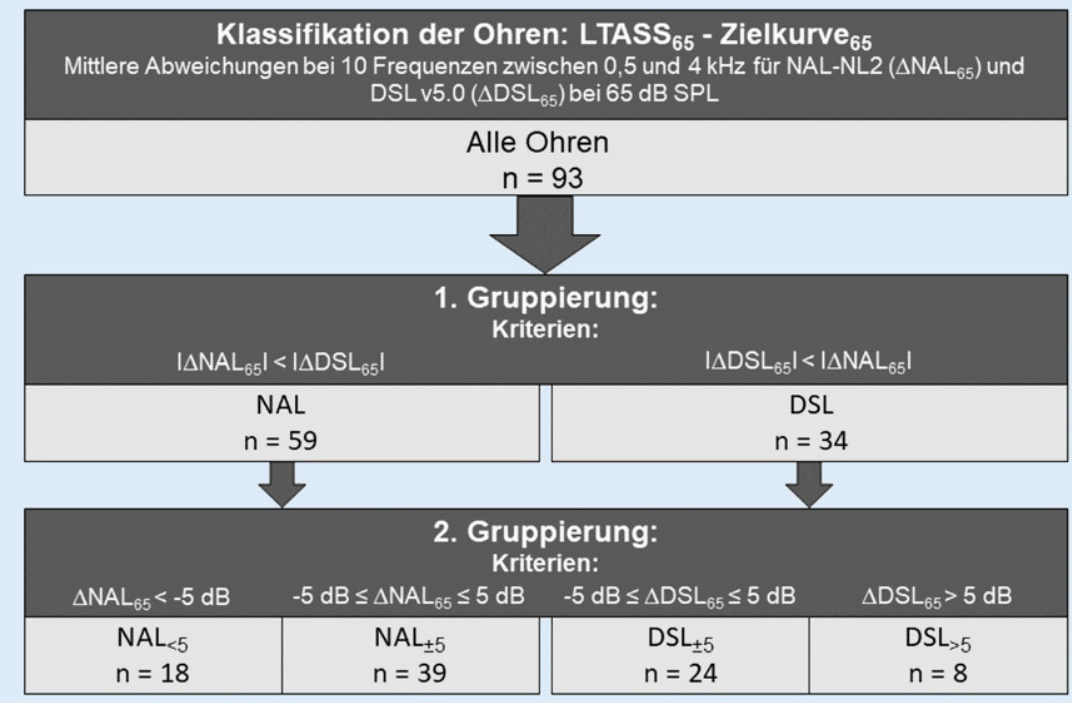

Abb. 1 \ Schematischer Aufbau der Gruppierungsvorgänge. (Abkürzungen siehe Verzeichnis)

tenohr vermessen. Hierzu wurde das ISTS mit 50, 65 und $80 \mathrm{~dB}$ SPL ( $\left.\mathrm{L}_{\mathrm{E}-50}, \mathrm{~L}_{\mathrm{E}-65}, \mathrm{~L}_{\mathrm{E}-80}\right)$ über $30 \mathrm{~s}$ dargeboten und die REAR aufgezeichnet. Aus den digitalen Messprotokollen heraus wurden für jedes Ohr folgende Parameter extrahiert:

1. Die Hör- und Unbehaglichkeitsschwellen wurden gemäß ANSI 3.6-2004 [2] von $\mathrm{dB} H \mathrm{HL}$ in $\mathrm{dB}$ SPL umgerechnet, um mit den REAR-Messungen verglichen werden zu können. Die Schwelle in SPL ergibt sich aus der Schwelle in HL mit Berücksichtigung des hörerspezifischen äquivalenten BezugsSchwellenschalldruckpegels („reference equivalent threshold sound pressure level", RETSPL) und der Differenz zwischen dem Schalldruckpegel im Ohr und dem im 6cc-Kuppler, welcher für die Kalibrierung verwendet wird ("real-ear to coupler difference ${ }^{\prime \prime}$ RECD): Hörschwelle [dB SPL] = Hörschwelle $[d B H L]+R^{2} C_{6 c c}+$ RETSPL, [2].

2. Aus den REAR-Messungen wurden für 20 Frequenzen $F_{n}$ in Terzabständen $\left(F_{n}=0,125 * 2^{(n / 3)} \mathrm{kHz}, n=0,1, \ldots, 19\right)$ jeweils die Terzpegel für das mittlere Langzeit-Sprach-Spektrum („long-term average speech spectrum", LTASS), das 30. Perzentil und das 99. Perzentil berechnet.

3. Die Zielkurven für das LTASS (REAR in dB SPL, Testsignal ISTS) wurden gemäß NAL-NL2 und DSL v5.0 für $L_{E-50} L_{E-65}$ und $\mathrm{L}_{\mathrm{E}-80}$ bestimmt. Die Zielkurvenberechnung basiert auf den Hörschwellen und weiteren Parametern wie z. B. Hörgeräteerfahrung, Otoplastik und vorhandene Zusatzbohrung. Für DSL v5.0 wird außerdem die gemessene Unbehaglichkeitsschwelle berücksichtigt. Bei hochgradigem Hörverlust kann es vorkommen, dass einige Zielwerte unterhalb der Hörschwellen liegen. Dies kann der geringen Restdynamik geschuldet sein und wird in der Praxis vor allem bei hochgradigem Hochtonhörverlust beobachtet [11].

4. Basierend auf den REAR-Messungen wurde der SII für $L_{E-50}, L_{E-65}$ und $L_{E-80}$ berechnet. Gemäß ANSI 3.5-1997 [1] wird zur Bestimmung des SII die Hörbarkeit ( 0 bis 1 ) für die 18 verwendeten Terzbänder zwischen 0,16 und $8 \mathrm{kHz}$ ermittelt. Zusätzlich erfolgt eine Gewichtung der einzelnen Terzbänder nach Relevanz (0 bis 1) für das jeweils verwendete Sprachmaterial. Durch das Aufsummieren des Produkts dieser Werte über die verschiedenen Frequenzbänder hinweg wird ein einzelner numerischer Index, der SII, erhalten. Der SII kann von 0 bis 1 oder in \% angegeben werden. Ein SII von 0 bedeutet also, dass keine Sprachanteile des jeweiligen Sprachmaterials hörbar sind, während ein SII von 1 eine vollständige Hörbarkeit bedeutet.

\section{Datenanalyse}

Die Datenanalyse erfolgte für jedes der 93 Ohren separat. Hierzu wurden die Daten aus dem Ton- und Sprachaudiogramm, den Freifeld- sowie In-situMessungen betrachtet. Zusätzlich wurde aus dem $\mathrm{EV}_{65}(\mathrm{HG})$ und dem $\mathrm{mEV}$ der Wirkungsgrad $\mathrm{Q}$ bestimmt. Die Analyse des Shapiro-Wilk-Tests zeigte, dass die erhobenen Daten nicht normalverteilt waren. Für die statistischen Vergleiche der Mediane von mehr als zwei unabhängigen Stichproben wurde daher der Kruskal-Wallis-Test verwendet. Die Posthoc-Analyse erfolgte mittels Dunn-Bonferroni-Tests. Das Signifikanzniveau betrug für alle Tests $a=0,5$. Für die Berechnung von Korrelationen fand das Verfahren nach Spearman Anwendung. Signifikante Unterschiede zwischen den Gruppen sind in den Grafiken durch ${ }^{*}(p<0,05)$, ${ }^{* *}(p<0,01)$ oder ${ }^{* *}(p<0,001)$ gekennzeichnet. Die statistischen Tests wurden mit dem Statistikprogramm Statistical Package for Social Sciences (SPSS V24, Fa. IBM Corp., Armonk, NY, USA) durchgeführt und die Abbildungen mit Matlab ${ }^{\circledR}$ R2020b (Fa. Mathworks, Natick, MA, USA) erstellt.

Die Daten für die einzelnen Ohren wurden basierend auf den Ergebnissen der In-situ-Messungen gruppiert (- Abb. 1). Hierzu wurden bei $\mathrm{L}_{\mathrm{E}-65}$ für jedes $\mathrm{Ohr}$ die mittleren Differenzen zwischen gemessenem LTASS des Ausgangssignals bei $65 \mathrm{~dB}$ SPL $\left(\right.$ LTASS $_{65}$ ) und den beiden Zielkurven für die zehn Frequenzen zwischen 0,5 und $4 \mathrm{kHz}$ bestimmt $\left(\triangle \mathrm{NAL}_{65}\right.$ und $\left.\Delta D L_{65}\right)$. Für $\left|\Delta N A L_{65}\right|<\left|\Delta D L_{65}\right|$ wurde das Ohr der NAL-Gruppe und für $\left|\Delta \mathrm{DSL}_{65}\right|<\left|\Delta \mathrm{NAL}_{65}\right|$ der DSL-Gruppe zugeordnet. Anschließend wurden die beiden Gruppen unterteilt, in solche mit sehr guter Übereinstimmung an die Zielkurven $\left(\mathrm{NAL}_{ \pm 5}, \mathrm{DSL}_{ \pm 5}\right)$ und in solche mit mittleren Abweichungen von mehr als $5 \mathrm{~dB}$ von den Zielkurven $\left(\mathrm{NAL}_{<5}, \mathrm{DSL}_{>5}\right.$ ).

Für die Parameter $\mathrm{EV}_{65}(\mathrm{HG})$ und $\mathrm{Q}$ wurden jeweils die Spearman-Korrelationskoeffizienten für den SII berechnet. Hierbei ist zu beachten, dass der SII unter Verwendung des ISTS bestimmt wurde. $\mathrm{EV}_{65}(\mathrm{HG})$ und $\mathrm{Q}$ hingegen wurden mittels des Freiburger Einsilbertests bestimmt, welcher eine andere spektrale Verteilung aufweist. 


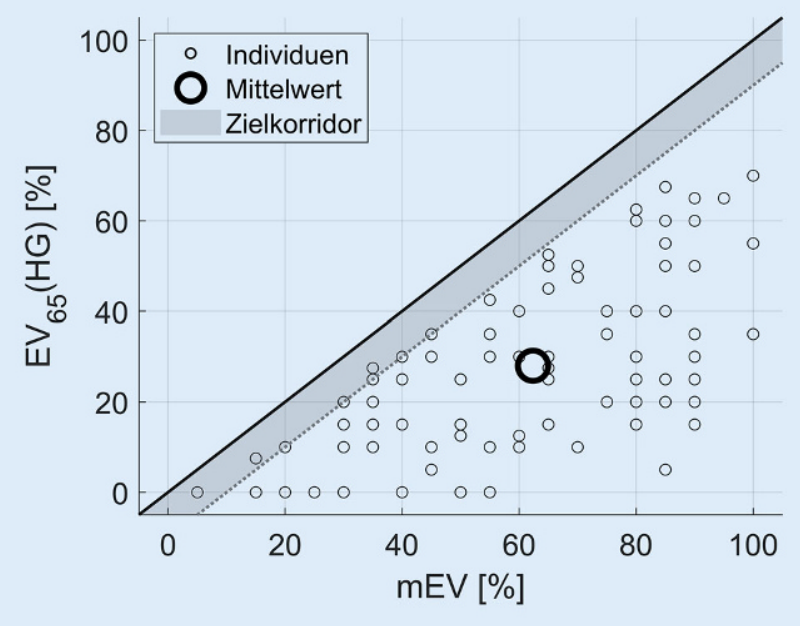

Ergebnisse

In $\triangle$ Abb. 2 ist das $\mathrm{EV}_{65}(\mathrm{HG})$ in Abhängigkeit vom $\mathrm{mEV}$ als Scatterplot dargestellt. Die graue Fläche markiert den Zielbereich für eine nach Kießling et al. [13] akzeptable Hörgeräteversorgung. Die Versorgungsergebnisse liegen größtenteils unterhalb des angestrebten Bereichs (91\%). Das $\mathrm{mEV}$ wurde in keinem Fall erreicht oder übertroffen. Das mittlere $\mathrm{EV}_{65}(\mathrm{HG})$ liegt 34,4\%-Punkte unter dem mittleren $\mathrm{mEV}$. Die nach der Hilfsmittel-Richtlinie [6] geforderte Verbesserung von mindestens $20 \%$-Punkten zeigte sich hingegen bei $65 \%$ der Messungen. Bei 10\% der Hörgeräteversorgungen wurde kein Gewinn erzielt.

In-situ-Messungen im Vergleich zu den Zielkurven

Die Ergebnisse der In-situ-Messungen sind für alle 93 Ohren als Mittelwerte in $\bullet$ Abb. 3 für NAL-NL2 in der linken Spalte und für DSL v5.0 in der rechten Spalte für niedrige ( $L_{E-50}$, oben), mittlere ( $L_{E-65}$, mittig) und hohe ( $\mathrm{L}_{\mathrm{E}-80}$, unten) Eingangspegel dargestellt. Der hörbare, weiß dargestellte Dynamikbereich ist nach unten durch die Hörschwelle und nach oben durch die Unbehaglichkeitsschwelle begrenzt.

- $L_{E-50}$ : Sowohl die Sprachanteile des Ausgangssignals als auch die beiden Zielkurven liegen fast vollständig unter der Hörschwelle. Lediglich die 99. Perzentilkurve liegt im Bereich
Abb. $2 \triangleleft$ Einsilberverstehen $(n=93$ Ohren) mit Hörgerät bei $65 \mathrm{~dB}$ SPL $\left(\mathrm{EV}_{65}(\mathrm{HG})\right)$ in $\mathrm{Ab}$ hängigkeit vom maximalen Einsilberverstehen (mEV). Graue Fläche Zielkorridor von max. $10 \%$ Diskrepanz zwischen $\mathrm{mEV}$ und $\mathrm{EV}_{65}(\mathrm{HG})$. SPL Schalldruckpegel, $d B$ Dezibel von 500 bis $1500 \mathrm{~Hz}$ leicht über der Hörschwelle.

- $L_{E-65}$ : Das LTASS liegt nur zwischen 0,5 und $2 \mathrm{kHz}$ oberhalb der Hörschwelle. Die Zielkurve für NAL-NL2 und DSL v5.0 unterscheiden sich hier deutlich: Die NAL-NL2-Zielkurve liegt auf oder leicht unterhalb der Hörschwelle, die DSLv5.0-Zielkurve liegt jedoch um bis zu $12 \mathrm{~dB}$ oberhalb der Hörschwelle. Im Mittel liegen alle Zielkurven für DSL v5.0 über den Zielkurven für NALNL2 (Mittelwert: 8,8 dB).

- $L_{E-80}$ : Die Zielkurven und das LTASS liegen im Bereich von 0,25 bis $3 \mathrm{kHz}$ auf oder oberhalb der Hörschwelle. Im Mittel liegen alle Zielkurve für DSL v5.0 über diejenigen für NAL-NL2 (Mittelwert: $14 \mathrm{~dB}$ ).

\section{Ausgangsschalldruckpegel für die einzelnen Verstärkungsgruppen}

Aus dem Vergleich von dem LTASS $_{65}$ und den Zielkurven bei $\mathrm{L}_{\mathrm{E}-65}$ wurden wie oben beschrieben vier Gruppen gebildet (- Abb. 1). Dabei war die Übereinstimmung zwischen Ziel und Messung bei 39 Ohren für NAL-NL2 $\left(\mathrm{NAL}_{ \pm 5}\right)$ und bei 24 Ohren für DSL v5.0 (DSL ${ }_{ \pm 5}$ ) am größten. Bei 18 Ohren lag das LTASS $_{65}$ deutlich niedriger als die NAL-NL2-Zielkurve $\left(\mathrm{NAL}_{<5}\right)$. Bei 8 Ohren lag das LTASS $_{65}$ deutlich über der DSL-v5.0-Zielkurve $\left(\mathrm{DSL}_{>5}\right)$. Es verblieben vier Ohren, welche keines der Kriterien aus Abb. 1 erfüllten. Hier lag das LTASS $_{65}$ mehr als $5 \mathrm{~dB}$ über NAL-NL2 und mehr als $5 \mathrm{~dB}$ unter DSL v5.0. Die
Differenzen zwischen LTASS und Zielkurve ( $\triangle \mathrm{NAL}(\mathrm{f})$ und $\triangle \mathrm{DSL}(\mathrm{f})$ ) sind in $\mathbf{A b b} \mathbf{4}$ für die Gruppen mit gut passender REAR ( $\mathrm{NAL}_{ \pm 5}$ links und $\mathrm{DSL}_{ \pm 5}$ rechts) dargestellt. Die - Abb. 5 zeigt die gleiche Ansicht für Gruppen mit deutlich niedrigerer $\left(\mathrm{NAL}_{<5}\right)$, bzw. deutlich höherer REAR (DSL $>5$ ).

Obwohl das LTASS $_{65}$ und die Zielkurve für die beiden Gruppen $\mathrm{NAL}_{ \pm 5}$ und $\mathrm{DSL}_{ \pm 5}$ bei $\mathrm{L}_{\mathrm{E}-65}$ sehr gut übereinstimmen, sind bei niedrigeren und höheren Eingangspegeln deutliche Abweichungen zu beobachten. Für $\mathrm{LE}_{\mathrm{E}-50}$ liegt das $\mathrm{LTASS}_{50}$ tendenziell über der Zielkurve für DSL v5.0 und unterhalb derjenigen für NAL-NL2. Bei $\mathrm{L}_{\mathrm{E}-80}$ liegt das LTASS $_{80}$ für NAL $\mathrm{N}_{ \pm 5}$ größtenteils oberhalb der Zielkurve und für $\mathrm{DSL}_{ \pm 5}$ unterhalb.

Die in -Abb. 5 dargestellten Differenzen zwischen LTASS und Zielkurve für $\mathrm{NAL}_{<5}$ (linke Spalte) und $\mathrm{DSL}_{>5}$ (rechte Spalte) zeigen, dass die Abweichungen für alle drei Eingangspegel zu finden sind. Die Abweichungen sind für $\mathrm{L}_{\mathrm{E}-50}$ am größten und nehmen mit zunehmendem Eingangspegel ab.

\section{Einsilberverstehen mit Hörgerät und Wirkungsgrad der Hörgeräte- versorgung in Abhängigkeit vom Sprachverständlichkeitsindex}

Die vier Gruppen $\left(\mathrm{NAL}_{<5}, \mathrm{NAL}_{ \pm 5}, \mathrm{DSL}_{ \pm 5}\right.$ und $D S L_{>5}$ ) werden durch unterschiedliche Symbole repräsentiert. Die schwarzen Symbole bilden jeweils die Mittelwerte der einzelnen Gruppen. Zusätzlich werden die vier restlichen Ohren gezeigt, bei denen keines der Gruppierungskriterien erfüllt wurde (Andere). Obwohl große Überschneidungen der Gruppensymbole auftreten, gibt es Unterschiede zwischen den Gruppen hinsichtlich der Parameter $\mathrm{EV}_{65}(\mathrm{HG}), \mathrm{Q}$ und SII. In DTab. 1 werden die Mediane und die Mittelwerte der einzelnen Parameter für die vier Gruppen dargestellt. Die Analyse des Kruskal-Wallis-Tests zeigte für $4 \mathrm{FPTA} \mathrm{EV}_{65}(\mathrm{HG}), \mathrm{Q}$ und SII einen hochsignifikanten Gruppeneffekt. Die Ergebnisse der Dunn-Bonferroni-Tests sind in - Tab. 2 aufgeführt.

In Abb. 6 wird der SIl jeweils mit dem $\mathrm{EV}_{65}(\mathrm{HG})$ (linke Spalte) und mit dem Wirkungsgrad Q (rechte Spalte) in Form von Scatterplots für alle 93 Ohren in Beziehung gesetzt. Trotz der großen Variabilität ergibt sich eine hochsignifikante Korrelati- 

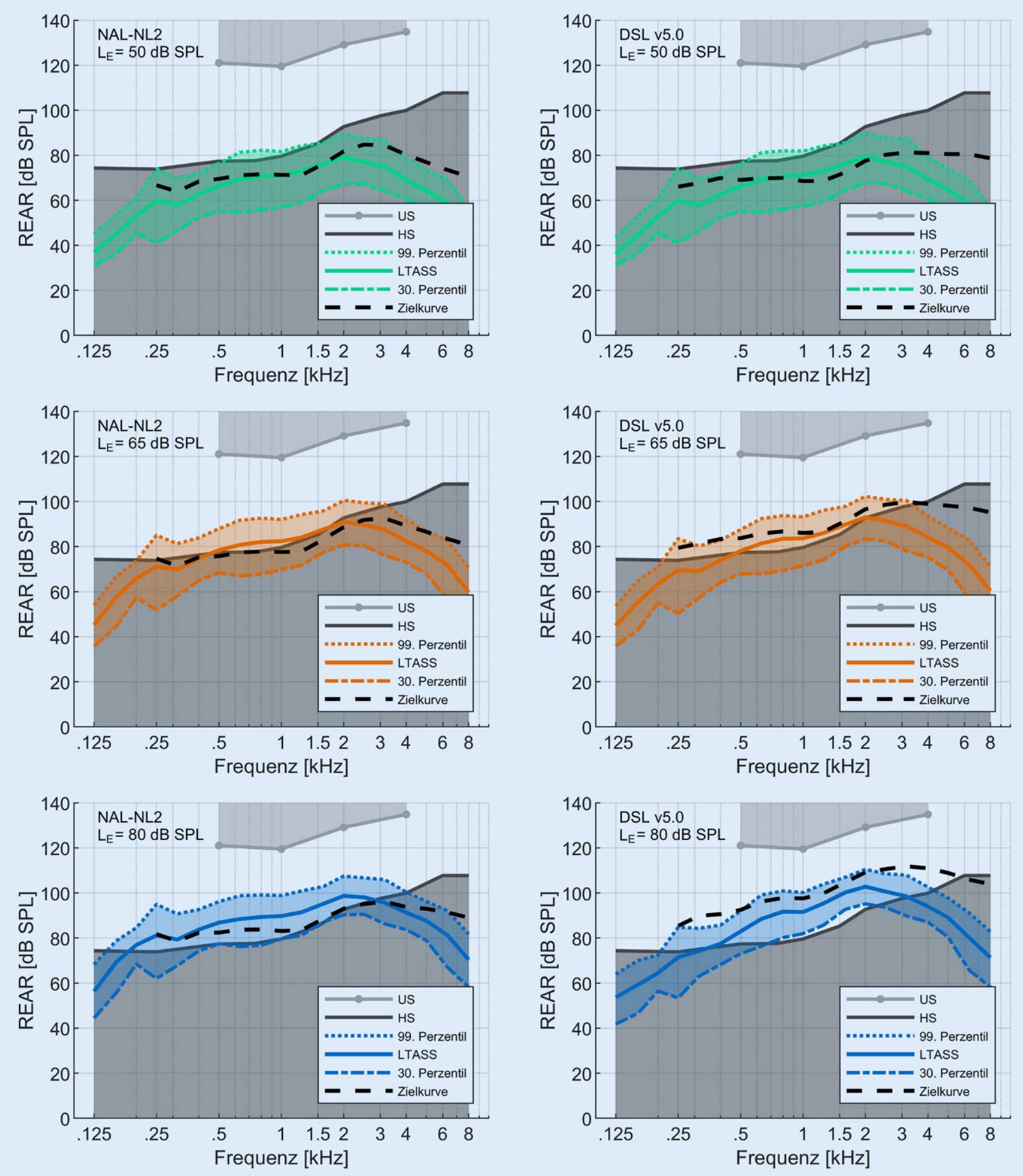

Abb. $3 \Delta$ Ausgangsschalldruckpegel am Trommelfell (REAR) als Funktion der Frequenz. Die obere hellgraue Begrenzung stellt die Unbehaglichkeitsschwelle (US) und die untere dunkelgraue Begrenzung die Hörschwelle (HS) dar. Die gestrichelte Linie (schwarz) repräsentiert die Zielkurve: NAL-NL-2 (linke Spalte), DSL v5.0 (rechte Spalte). Die durchgezogene farbige Linie bildet das LTASS, und die gestrichelten farbigen Linien bilden das 30. (untere Linie) bzw. das 99. (obere Linie) Perzentil nach. Die Eingangspegel waren $50 \mathrm{~dB}$ SPL (grün), 65 dB SPL (orange) und $80 \mathrm{~dB}$ SPL (blau). Gezeigt werden die Mittelwerte über alle Ohren ( $n=93$ Ohren). (Abkürzungen siehe Verzeichnis) 

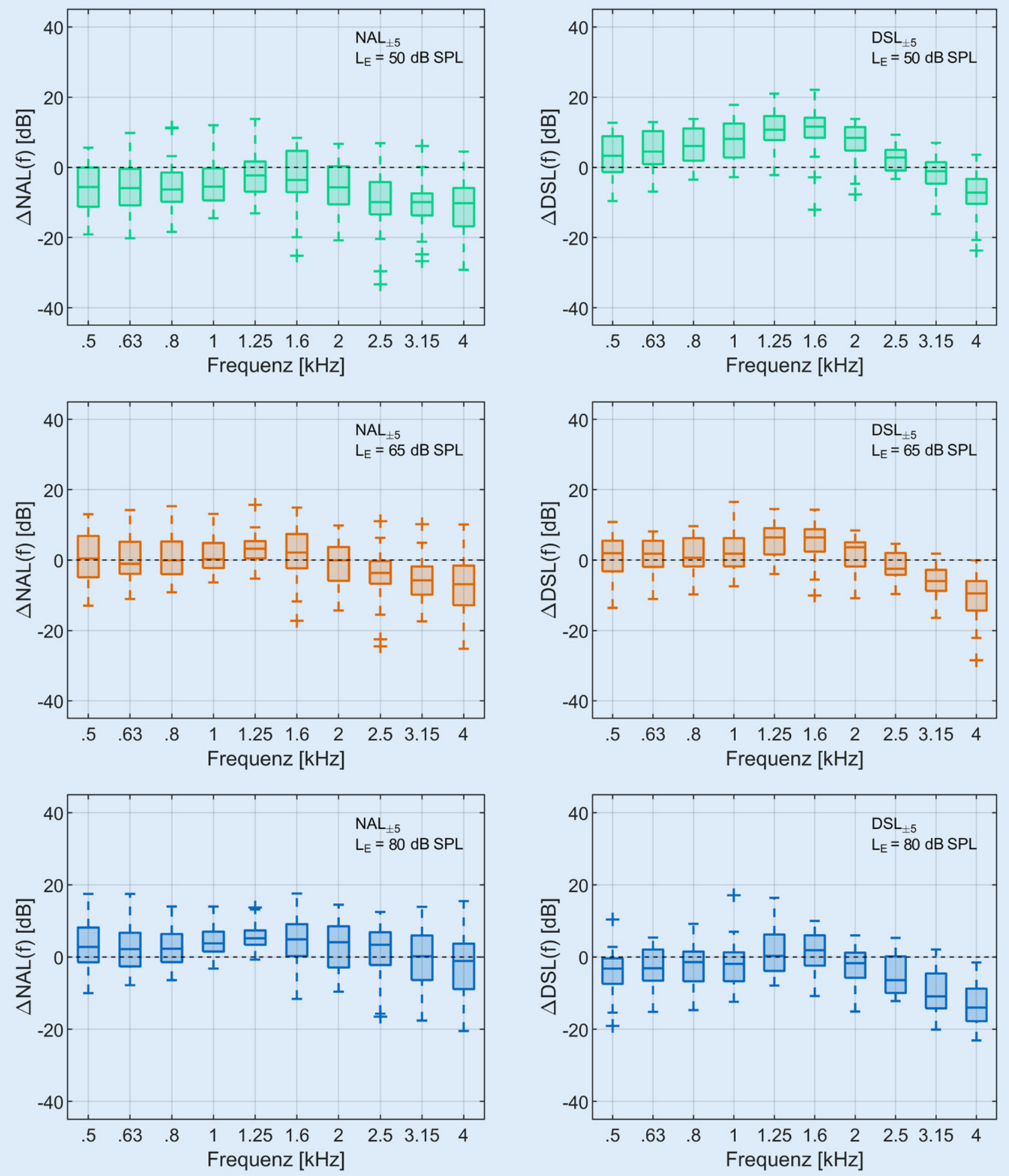

Abb. $4 \Delta$ Differenzzwischen LTASS und Zielkurve fürNAL-NL2 $(\triangle N A L(f), n=39)$ in der linken Spalte und fürDSL v5.0 ( $\triangle \mathrm{DSL}(\mathrm{f})$ $n=24)$ in der rechten Spalte für $\mathrm{NAL}_{ \pm 5}$ und $\mathrm{DSL}_{ \pm 5}$ im Frequenzbereich von $0,5-4 \mathrm{kHz}$. Boxplots Median (-), Interquartilsabstand (Boxlänge), Whiskers (maximal das 1,5-Fache des Interquartilsabstands) und Ausreißer (+) Die Eingangspegel waren $50 \mathrm{~dB}$ SPL (grün), $65 \mathrm{~dB}$ SPL (orange) und $80 \mathrm{~dB}$ SPL (blau). (Abkürzungen siehe Verzeichnis) 

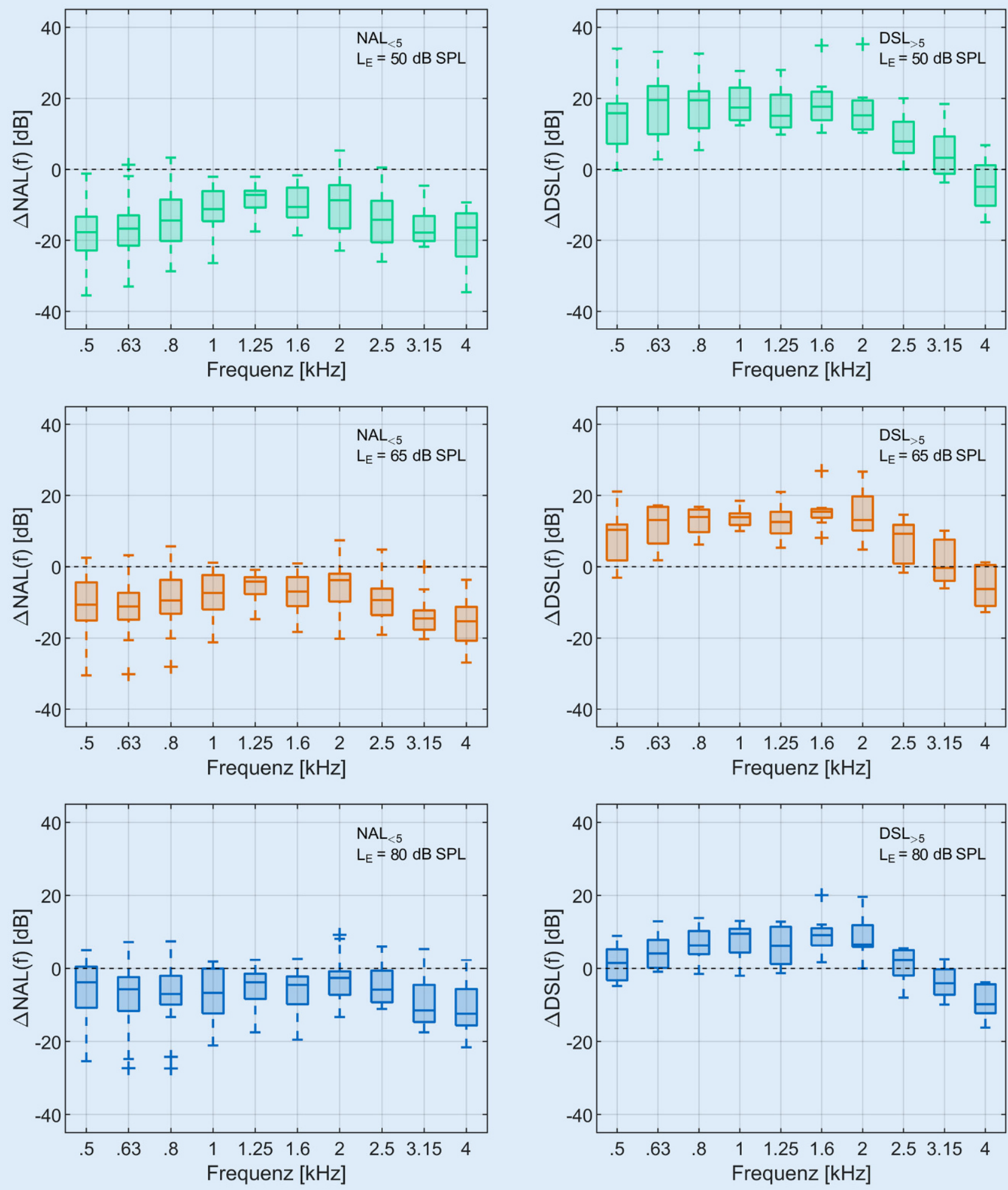

Abb. $5 \Delta$ Differenzzwischen LTASS und Zielkurve fürNAL-NL2 $(\triangle N A L(f), n=18)$ in der linken Spalte und fürDSL v5.0 ( $\triangle \mathrm{DSL}(\mathrm{f})$ $n=8$ ) in der rechten Spalte für $\mathrm{NAL}_{<5}$ und $\mathrm{DSL}_{>5}$ im Frequenzbereich von $0,5-4 \mathrm{kHz}$. Boxplots Median $(-)$, Interquartilsabstand (Boxlänge), Whiskers (maximal das 1,5-Fache des Interquartilsabstands) und Ausreißer (+). Die Eingangspegel waren $50 \mathrm{~dB}$ SPL (grün), $65 \mathrm{~dB}$ SPL (orange) und $80 \mathrm{~dB}$ SPL (blau). (Abkürzungen siehe Verzeichnis) 

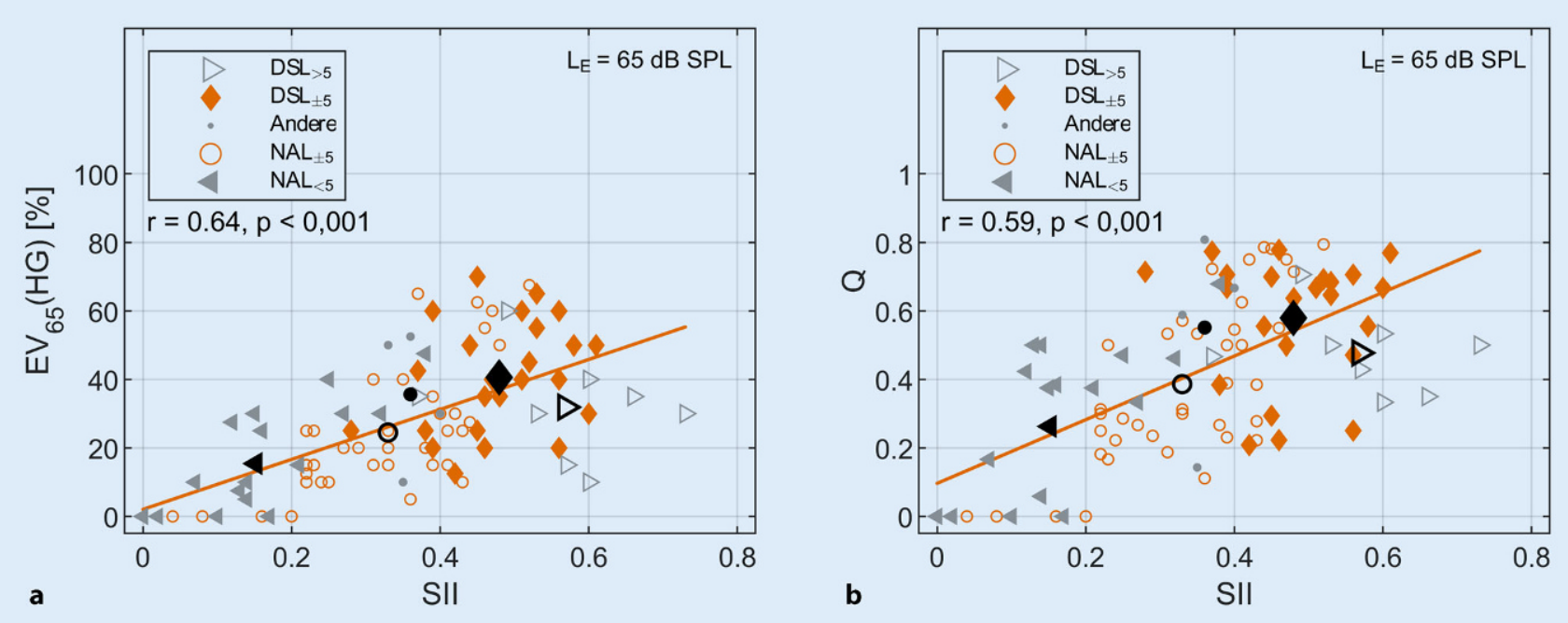

Abb. 6 \ Scatterplot und Korrelationsanalyse ( $n=93$ Ohren). (a) Einsilberverstehen mit Hörgerät bei $65 \mathrm{~dB} \mathrm{SPL}\left(\mathrm{EV}_{65}(\mathrm{HG})\right)$ und Sprachverständlichkeitsindex (SII). (b) Wirkungsgrad Q und SII. Zusätzlich sind die Regressionsgeraden (orange) eingezeichnet. Den verschiedenen Gruppen wurden unterschiedliche Symbole zugeordnet: $\mathrm{NAL}_{<5}$ (Dreieck, nach links zeigend), $\mathrm{NAL}_{ \pm 5}$ (Kreis), $\mathrm{DSL}_{ \pm 5}$ (Diamant), DSL>5 (Dreieck, nach rechts zeigend) und Andere (Punkt). Die schwarzen Symbole bilden den Mittelwert der einzelnen Gruppen. (Abkürzungen siehe Verzeichnis)

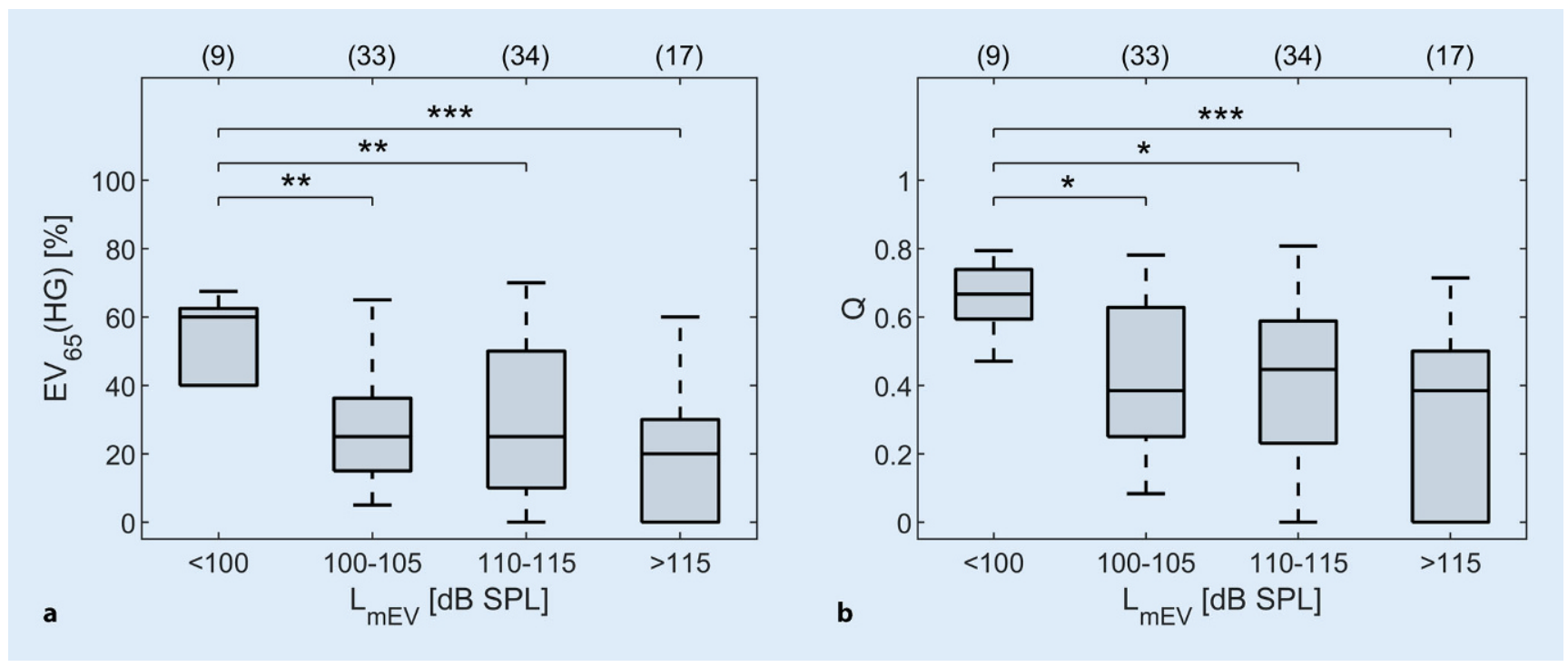

Abb. 7 \ Einsilberverstehen mit Hörgerät bei $65 \mathrm{dBSPL}\left(\mathrm{EV}_{65}(\mathrm{HG})\right.$, a) und Wirkungsgrad (Q, b) in Abhängigkeit vom Pegel für das maximale Einsilberverstehen ( $L_{\mathrm{mEV}}$ ). Boxplots Median (-), Interquartilsabstand (Boxlänge) und Whiskers (maximal das 1,5-Fache des Interquartilsabstands). Asteriske In der Post-hoc-Analyse festgestellte Signifikanzniveaus, $p<0,05\left(^{*}\right), p<0,01$ ${ }^{(* *)}$ und $p<0,001\left(^{* * *}\right) . d B$ Dezibel, SPL Schalldruckpegel

on von $\mathrm{r}=0,64(p<0,001)$ für $\mathrm{EV}_{65}(\mathrm{HG})$ und $r=0,59(p<0,001)$ für $\mathrm{Q}$. Der Mittelwert und die Standardabweichung betragen jeweils für $\mathrm{EV}_{65}(\mathrm{HG}) 28 \pm 19,1 \%$ und für $\mathrm{Q}$ $0,43 \pm 0,24$.
Einfluss des Schallpegels für das maximale Einsilberverstehen auf das Einsilberverstehen mit Hörgerät und den Wirkungsgrad der Hörgeräteversorgung

Alle 93 Ohren wurden in vier Gruppen eingeordnet, um den Effekt des Pegels für das maximale Einsilberverstehen ( $\left.L_{m E v}\right)$ auf $\mathrm{EV}_{65}(\mathrm{HG})$ und $\mathrm{Q}$ zu untersuchen. In
- Abb. 7 werden die Ohren in Boxplots wie folgt zusammengefasst: $L_{m E v}<100 \mathrm{~dB} S P L$ (G1), $L_{m E V}=100-105 d B$ SPL (G2), $L_{m E V}=$ 110-115 dB SPL (G3) und $L_{m E V}>115 \mathrm{~dB} S P L$ (G4). Die Mediane betragen für $\mathrm{EV}_{65}(\mathrm{HG})$ $60 \%(\mathrm{G} 1), 25 \%(\mathrm{G} 2, \mathrm{G} 3)$ und $20 \%(\mathrm{G} 4)$ und für $Q \quad 0,67(G 1), 0,38(G 2), 0,45(G 3)$ und 0,38 (G4). Für die beiden Parameter $\mathrm{EV}_{65}(\mathrm{HG})$ und $\mathrm{Q}$ konnte ein signifikanter Gruppeneffekt gefunden werden 
Tab. 1 Verstärkungsgruppen $\mathrm{NAL}_{<5}, \mathrm{NAL}_{ \pm 5}, \mathrm{DSL}_{ \pm 5}$ und $\mathrm{DSL}_{>5}$ mit dazugehörigen Median- und Mittelwerten für die einzelnen Parameter

\begin{tabular}{|c|c|c|c|c|c|c|c|c|}
\hline- & \multicolumn{2}{|l|}{$\mathrm{NAL}_{<5}$} & \multicolumn{2}{|l|}{$\mathrm{NAL}_{ \pm 5}$} & \multicolumn{2}{|l|}{$\mathrm{DSL}_{ \pm 5}$} & \multicolumn{2}{|l|}{$\mathrm{DSL}_{>5}$} \\
\hline Anzahl & \multicolumn{2}{|l|}{18} & \multicolumn{2}{|l|}{39} & \multicolumn{2}{|l|}{24} & \multicolumn{2}{|l|}{8} \\
\hline $\begin{array}{l}\operatorname{Min} / \max \\
\Delta \mathrm{NAL}_{65} / \Delta \mathrm{DSL}_{65}[\mathrm{~dB}]\end{array}$ & \multicolumn{2}{|l|}{$-22 /-5,3$} & \multicolumn{2}{|l|}{$-4,8 / 3,9$} & \multicolumn{2}{|l|}{$-4,8 / 4,7$} & \multicolumn{2}{|l|}{$5,1 / 14,2$} \\
\hline- & Median & $\begin{array}{l}\text { Mittel- } \\
\text { wert }\end{array}$ & Median & $\begin{array}{l}\text { Mittel- } \\
\text { wert }\end{array}$ & Median & $\begin{array}{l}\text { Mittel- } \\
\text { wert }\end{array}$ & Median & $\begin{array}{l}\text { Mittel- } \\
\text { wert }\end{array}$ \\
\hline Alter [Jahre] & 65 & 65,4 & 68 & 65,9 & 69 & 65,3 & 78 & 70 \\
\hline 4FPTA [dB HL] & 81,6 & 81,2 & 68,3 & 72,1 & 67,3 & 69,3 & 69,9 & 71,4 \\
\hline $\mathrm{L}_{\mathrm{mEv}}[\mathrm{dB} S \mathrm{SPL}]$ & 110 & 108,6 & 100 & 105 & 110 & 106,3 & 110 & 112,5 \\
\hline $\mathrm{mEV}[\%]$ & 57,5 & 53,6 & 55 & 59,7 & 80 & 71,7 & 67,5 & 65 \\
\hline $\mathrm{EV}_{65}[\%]$ & 0 & 2,2 & 0 & 2,7 & 0 & 3,1 & 0 & 0 \\
\hline $\mathrm{EV}_{65}(\mathrm{HG})[\%]$ & 10 & 15,4 & 20 & 24,5 & 40 & 40,6 & 32,5 & 31,9 \\
\hline Q & 0,36 & 0,26 & 0,31 & 0,39 & 0,67 & 0,58 & 0,48 & 0,48 \\
\hline $\mathrm{SII}_{65}$ & 0,14 & 0,15 & 0,35 & 0,33 & 0,48 & 0,48 & 0,59 & 0,57 \\
\hline
\end{tabular}

Tab. 2 Vergleich derVerstärkungsgruppen $\mathrm{NAL}_{<5}, \mathrm{NAL}_{ \pm 5}, \mathrm{DSL}_{ \pm 5}$ und $\mathrm{DSL}_{>5}$ : Ergebnisse der Kruskal-Wallis-Tests (Gruppeneffekt) und der Dunn-Bonferroni-Tests (Paarvergleich) für die einzelnen Parameter

\begin{tabular}{|c|c|c|c|c|c|c|c|}
\hline- & $\begin{array}{l}\text { Kruskal- } \\
\text { Wallis-Test }\end{array}$ & $\begin{array}{l}\mathrm{NAL}_{<5} / \\
\mathrm{NAL}_{ \pm 5}\end{array}$ & $\begin{array}{l}\mathrm{NAL}_{<5} / \\
\mathrm{DSL}_{ \pm 5}\end{array}$ & $\begin{array}{l}\mathrm{NAL}_{<5} / \\
\mathrm{DSL}_{>5}\end{array}$ & $\begin{array}{l}\mathrm{NAL}_{ \pm 5} / \\
\mathrm{DSL}_{ \pm 5}\end{array}$ & $\begin{array}{l}\mathrm{NAL}_{ \pm 5} / \\
\mathrm{DSL}_{>5}\end{array}$ & $\begin{array}{l}\mathrm{DSL}_{ \pm 5} / \\
\mathrm{DSL}_{>5}\end{array}$ \\
\hline Alter & $\begin{array}{l}X^{2}(3)=0,791 \\
p=0,852\end{array}$ & - & - & - & - & - & - \\
\hline 4FPTA* & $\begin{array}{l}x^{2}(3)=10,711 \\
p=0,013\end{array}$ & $p=0,084$ & $p=0,009$ & $p=0,144$ & $p=1,000$ & $p=1,000$ & $p=1,000$ \\
\hline$L_{m E V}$ & $\begin{array}{l}X^{2}(3)=6,332 \\
p=0,097\end{array}$ & - & - & - & - & - & - \\
\hline $\mathrm{mEV}$ & $\begin{array}{l}X^{2}(3)=6,856 \\
p=0,077\end{array}$ & - & - & - & - & - & - \\
\hline $\mathrm{EV}_{65}$ & $\begin{array}{l}X^{2}(3)=3,014 \\
p=0,389\end{array}$ & - & - & - & - & - & - \\
\hline $\mathrm{EV}_{65}(\mathrm{HG})^{*}$ & $\begin{array}{l}X^{2}(3)=20,797 \\
p<0,001\end{array}$ & $p=0,714$ & $p<0,001$ & $p=0,155$ & $p=0,004$ & $p=1,000$ & $p=1,000$ \\
\hline$Q^{*}$ & $\begin{array}{l}X^{2}(3)=18,069 \\
p<0,001\end{array}$ & $p=0,559$ & $p<0,001$ & $p=0,311$ & $p=0,014$ & $p=1,000$ & $p=1,000$ \\
\hline $\mathrm{SIII}_{65}{ }^{*}$ & $\begin{array}{l}x^{2}(3)=55,115 \\
p<0,001\end{array}$ & $p=0,004$ & $p<0,001$ & $p<0,001$ & $p<0,001$ & $p=0,001$ & $p=1,000$ \\
\hline
\end{tabular}

Signifikante Gruppeneffekte werden durch Asterisk und signifikante Unterschiede zwischen den einzelnen Gruppen durch kursiv markierte Zellen gekennzeichnet $(a=0,05)$

(Abkürzungen siehe Verzeichnis)

Tab. 3 Vergleich der Gruppen G1 ( $\mathrm{L}_{\mathrm{mEV}}<100 \mathrm{~dB}$ SPL), G2 ( $\mathrm{L}_{\mathrm{mEV}}=100-105 \mathrm{~dB}$ SPL), G3 $\left(L_{m E V}=110-115 \mathrm{~dB} S P L\right)$ und G4 ( $\mathrm{L}_{\mathrm{mEV}}>115 \mathrm{~dB}$ SPL): Ergebnisse der Kruskal-Wallis-Tests (Gruppeneffekt) und der Dunn-Bonferroni-Tests (Paarvergleich) für die einzelnen Parameter

\begin{tabular}{|l|l|l|l|l|l|l|l|}
\hline- & $\begin{array}{l}\text { Kruskal- } \\
\text { Wallis-Test }\end{array}$ & $\mathbf{G 1 / G 2}$ & $\mathbf{G 1 / G 3}$ & $\mathbf{G 1 / G 4}$ & $\mathbf{G 2} / \mathbf{G 3}$ & $\mathbf{G 2} / \mathbf{G 4}$ & $\mathbf{G 3 / G 4}$ \\
\hline $\mathrm{EV}_{65}(\mathrm{HG})^{*}$ & $\begin{array}{l}\mathrm{X} 2(3)=15,394 \\
p=0,002\end{array}$ & $p=0,001$ & $p=0,009$ & $p=0,009$ & $p=1,000$ & $p=1,000$ & $p=1,000$ \\
\hline $\mathrm{Q}^{*}$ & $\begin{array}{l}\mathrm{X} 2(3)=11,428 \\
p=0,010\end{array}$ & $p=0,049$ & $p=0,038$ & $p=0,005$ & $p=1,000$ & $p=1,000$ & $p=1,000$ \\
\hline
\end{tabular}

Signifikante Gruppeneffekte werden durch Asterisk und signifikante Unterschiede zwischen den einzelnen Gruppen durch kursiv markierte Zellen gekennzeichnet $(a=0,05)$

$d B$ Dezibel, SPL Schalldruckpegel, $L_{m E V}$ Pegel beim maximalen Verstehen im Freiburger Einsilbertest, $E V_{65}(H G)$ Einsilberverstehen in Ruhe bei $65 \mathrm{~dB}$ SPL im Freifeld mit Hörgerät, $Q$ Wirkungsgrad
(• Tab. 3). Die Dunn-Bonferroni-Tests ergaben, dass die Patienten in der G1-Gruppe mit einem $L_{m E v}$ von $<100 \mathrm{~dB}$ ein signifikant besseres $\mathrm{EV}_{65}(\mathrm{HG})$ bzw. Q erreichten, als die Patienten in den anderen Gruppen (- Tab. 3). Zwischen den Gruppen G2, G3 und G4 gab es keine signifikanten Unterschiede. Für G2 bis G4 ist außerdem eine größere Variabilität der Daten zu erkennen.

\section{Diskussion}

In dieser Arbeit wurden sprachaudiometrische Größen in Bezug zu am Trommelfell anliegenden Sprachpegeln gesetzt. Im Fokus standen dabei das Sprachverstehen mit Hörgerät in Ruhe und dessen Beziehung zum maximalen Einsilberverstehen. Weiterhin wurde untersucht, ob das mit Hörgerät erreichte Sprachverstehen von der Abweichung der Hörgeräteverstärkung zu gängigen Zielvorgaben abhängt. In 63 Fällen $(67,7 \%)$ wurden die Zielkurven für NAL-NL2 oder DSL v5.0 bei $65 \mathrm{~dB}$ SPL mit Abweichungen von $\pm 5 \mathrm{~dB}$ zwar erreicht, dennoch liegt das $\mathrm{EV}_{65}(\mathrm{HG})$ im Mittel um 34,4\%-Punkte unter dem $\mathrm{mEV}$. Der mittlere Wirkungsgrad $\mathrm{Q}$ liegt bei 0,43 . Somit beträgt das Sprachverstehen mit Hörgerät im Mittel weniger als die Hälfte des mEV. Dies ist vergleichbar mit den Ergebnissen einer früheren Arbeit [5]. Für die 93 untersuchten Ohren nahmen sowohl $\mathrm{Q}$ als auch $\mathrm{EV}_{65}(\mathrm{HG})$ mit zunehmenden SII zu. Da die Zielkurven der mittleren und hohen Eingangspegel für DSL v5.0 über denjenigen für NAL-NL2 lagen, wurden dementsprechend auch bessere Werte für diejenige Ohren gefunden, bei denen das LTASS $_{65}$ näher an der entsprechenden Zielkurve für DSL v5.0 lag.

Sprachaudiogramm und Sprachverstehen mit Hörgerät

Im vorliegenden Patientenkollektiv liegt das $\mathrm{mEV}$ zwischen 5 und $100 \%$. Das $\mathrm{EV}_{65}(\mathrm{HG})$ liegt zwischen 0 und $70 \%$. Die mittlere Differenz zwischen $\mathrm{mEV}$ und $\mathrm{EV}_{65}(\mathrm{HG})$ beträgt 34,4\%-Punkte. Das sind ca. $15 \%$-Punkte mehr, als früher für ein großes Kollektiv mit unterschiedlichen Schwerhörigkeitsgraden beschrieben wurde [9]. Bei acht Ohren (8,6\%) liegen diese Differenzen im geforderten Bereich zwischen 5\%-Punkten $[19,20]$ und $10 \%$ - 
Punkten [13], allerdings wird das mEV in keinem Fall erreicht oder übertroffen. In der Literatur erfüllten 19 [14] bis $23 \%$ [9] diese Forderungen. Die Vorgabe aus der Hilfsmittel-Richtlinie (Verbesserung um mindestens $20 \%$-Punkte [6]) wurde in 60 Fällen $(64,5 \%)$ erfüllt. Das sind im Vergleich zur Literatur ca. 20\%-Punkte mehr [14]. Die Unterschiede könnten darin begründet sein, dass in dieser Arbeit ausschließlich Hörgeräteträger mit hochgradiger bis an Taubheit grenzendem Hörverlust ausgewertet wurden. Der mittlere Hörverlust liegt mit $72,9 \mathrm{~dB} \mathrm{HL}$ deutlich höher als in den zitierten Studien $[9,14]$.

Um den Wirkungsgrad Q zu bestimmen, wurde der Quotient aus den Parametern $\mathrm{EV}_{65}(\mathrm{HG})$ und $\mathrm{mEV}$ gebildet. Dieser gibt den Anteil am $\mathrm{mEV}$ an, welcher mit dem Hörgerät bei $65 \mathrm{~dB}$ SPL umgesetzt werden kann [5], beinhaltet allerdings keine Informationen über die Verbesserung des Sprachverstehens durch das Hörgerät. In diesem Patientenkollektiv befanden sich allerdings nur Patienten mit einem 4FPTA $\geq 60 \mathrm{~dB} \mathrm{HL}$ auf dem Hörgeräteohr, und das $\mathrm{EV}_{65}$ lag größtenteils bei $0 \%$. Ein $\mathrm{Q}>0$ entspricht somit in den meisten Fällen bereits einer Verbesserung durch das Hörgerät.

Für die Bewertung bei einzelnen Patienten ist der Wirkungsgrad $\mathrm{Q}$ allerdings kritisch zu interpretieren, da er ein Quotient aus zwei fehlerbehafteten Größen ist. Mittels Fehlerfortpflanzung auf Grundlage der Reliabilitäts-Daten von Winkler et al. 2016 [24] kann die Unsicherheit des Q-Werts geschätzt werden. Für die beiden typischen Werte $\mathrm{mEV}=60 \%$ und $\mathrm{EV}_{65}(\mathrm{HG})=30 \%$ ergibt sich zum Beispiel ein Q-Wert von 0,5 mit einer hohen Unsicherheit von $\pm 0,38$. Für die 93 Ohren liegt diese Unsicherheit im Mittel bei $\pm 0,31$. Zur Bewertung des Patientenkollektivs ist der Q-Wert dennoch geeignet, da aufgrund der relativ großen Anzahl an Messungen die Unsicherheit deutlich kleiner wird. So liegt diese für den mittleren Q-Wert aller Ohren bei lediglich $\pm 0,03$.

\section{In-situ-Messungen}

In 0 Abb. 3 sind die Ergebnisse der In-situMessungen für die präskriptiven Anpassformeln NAL-NL2 und DSL v5.0 dargestellt. Für $L_{E-50}$ ist der Großteil des Sprachsignals für die Patienten unhörbar (Mittelwert und Standardabweichung SII: 0,16 $\pm 0,12$ ). Der mittlere SII steigt bei $L_{E-65}$ auf $0,37 \pm 0,16$ und wird bei $\mathrm{L}_{\mathrm{E}-80}$ maximal $(0,48 \pm 0,16)$. Für $L_{E-65}$ und $L_{E-80}$ liegen im Mittel alle berechneten Zielkurven für DSL v5.0 über den Zielkurven für NAL-NL2. Somit wird für mittlere und hohe Eingangspegel bei DSL v5.0 ein höherer SIl erreicht als bei NAL-NL2. Hier stellt sich die Frage, inwieweit die Verstärkung für $L_{E-50}$ erhöht werden kann, um die Hörbarkeit für leise Sprachanteile wiederherzustellen. Die Maximierung der Hörbarkeit kann dabei nicht völlig unabhängig von den unterschiedlichen Eingangspegeln erfolgen. So lässt sich beispielsweise die Verstärkung für $\mathrm{L}_{\mathrm{E}-50}$ meist nicht beliebig erhöhen, sondern nur solange, bis eine ggf. hardwarebedingte, maximale Kompression erreicht wird (z. B. 3:1 für ein bestimmtes Hörgerät). Bei einer weiteren Erhöhung der Verstärkung für $\mathrm{L}_{\mathrm{E}-50}$, wird dann automatisch auch die Verstärkung fürhöhere Eingangspegel erhöht, also in diesem Beispiel für $\mathrm{L}_{\mathrm{E}-65}$. Die Verbesserung der Hörbarkeit für $\mathrm{L}_{\mathrm{E}-50}$ kann somit auch eine deutliche Erhöhung der Verstärkung für $\mathrm{L}_{\mathrm{E}-65}$ und $\mathrm{L}_{\mathrm{E}-80}$ mit sich bringen. Daher kommt zusätzlich die Frage auf, wie stark ggf. die Verstärkung für $\mathrm{L}_{\mathrm{E}-65}$ und $\mathrm{L}_{\mathrm{E}-80}$ erhöht werden kann, ohne die Akzeptanz der Einstellung zu beeinträchtigen. Eventuell kann es sich hier um konkurrierende Ziele in der Hörgeräteanpassung handeln, welche im gegenseitigen Widerspruch stehen, der nicht einfach aufgelöst werden kann. Das Zusammenspiel dieser möglichen Effekte müssten in weiteren Studien systematisch untersucht werden. Bis dahin muss für jeden Hörgeräteträger individuell abgewogen werden, inwieweit entweder eine höhere Verstärkung aller Eingangspegel oder eine Maximierung von $\mathrm{L}_{\mathrm{E}-50}$, ohne dabei die Verstärkung für höhere Eingangspegel zu verändern, sinnvoll und umsetzbar ist.

Hörgeräteevaluierung mittels Parameter aus der Sprachaudiometrie und den In-situ-Messungen

Ein weiteres Ziel der vorliegenden Arbeit war die Untersuchung des Zusammenhangs zwischen $\mathrm{EV}_{65}(\mathrm{HG})$ bzw. $\mathrm{Q}$ und der Annäherung an die präskriptiven Anpassformeln. Die 93 Hörgeräteeinstellungen wurden hierzu den vier Gruppen $\mathrm{NAL}_{<5}$, $\mathrm{NAL}_{ \pm 5}, \mathrm{DSL}_{ \pm 5}$ und $\mathrm{DS} \mathrm{L}_{>5}$ zugeordnet, entsprechend den mittleren absoluten Abweichungen zwischen LTASS $_{65}$ und der Zielkurve für NAL-NL2 bzw. DSL v5.0 bei $65 \mathrm{~dB}$ SPL im Hauptsprachbereich zwischen 0,5 und 4 kHz. Das LTASS 65 liegt bei $61,3 \%$ der Fälle nahe NAL-NL2 $( \pm 5 \mathrm{~dB})$ oder darunter. Bei $34,4 \%$ der Hörgeräteeinstellungen liegt das LTASS $_{65}$ nahe DSL v5.0 $( \pm 5 \mathrm{~dB})$ oder darüber. Bei den restlichen 4,3\% fand keine Gruppierung und statistische Analyse statt, da das LTASS $_{65}$ im Bereich zwischen den beiden Zielkurven liegt, aber keines der Gruppierungskriterien erfüllt wurde.

Die Werte der einzelnen Parameter sind für alle vier Gruppen in 0 Tab. 1 dargestellt. In $\mathrm{DSL}_{ \pm 5}$ waren die Ergebnisse mit Hörgerät, repräsentiert durch die Parameter $\mathrm{EV}_{65}(\mathrm{HG}), \mathrm{Q}$ und SIl, signifikant höher als in $\mathrm{NAL}_{ \pm 5}$ und $\mathrm{NAL}_{<5}$, obwohl die Voraussetzungen, repräsentiert durch $m E V, L_{m E V}$ und $\mathrm{EV}_{65}$, weitgehend gleich waren.

Die Gruppe mit der niedrigsten REAR im Vergleich zu den Zielkurven $\left(\mathrm{NAL}_{<5}\right)$ hatte auch die schlechtesten Hörergebnisse. Allerdings waren auch die Hörverluste in dieser Gruppe mit einem mittleren 4FPTA von $81,2 \mathrm{~dB}$ HL am höchsten. Auf der Basis der vorliegenden Daten kann nicht geklärt werden, ob eine größere Verstärkung durch technische oder psychoakustische Gründe verhindert wurde. Die höchsten REARs sind in der Gruppe $\mathrm{DSL}_{>5}$ zu finden. Hier liegen auch die SII-Werte deutlich höher. Allerdings ist die höhere REAR und Hörbarkeit nicht mit einem besseren Sprachverstehen verbunden. Im Vergleich $\mathrm{zu} \mathrm{DSL}_{ \pm 5}$ wurden keine signifikant höheren Werte für $\mathrm{EV}_{65}(\mathrm{HG}), \mathrm{Q}$ und SIl gefunden. Somit ist unklar, ob für eine Verstärkung für $L_{E-65}$, welche über die Präskription von DSL v5.0 hinausgeht, weitere Verbesserungen in $\mathrm{EV}_{65}(\mathrm{HG})$ bzw. Q zu erwarten sind. Ob eine Erhöhung der Verstärkung für niedrige Pegel (bzw. eine kompressivere Anpassstrategie) mit einer besseren Annäherung an das $m E V$ einhergeht, bleibt in weiteren Studien zu untersuchen.

Der SIl und die Verständlichkeit für Freiburger Einsilber korrelieren signifikant mit 0,64 . Höhere Werte sind schon allein deshalb nicht zu erwarten, da die SII-Berechnung durch die kommerzielle Software auf dem ISTS basiert und dementsprechende spezifische Frequenzgewichtungen ver- 
wendet. Daraus resultieren Abweichungen, die jedoch weit unterhalb der Bestimmungsfehler für die Sprachverständlichkeit liegen, was a posteriori durch die hohe Korrelation bestätigt wird.

\section{Grenzen des Versorgungserfolgs}

Neben dem maximalen Einsilberverstehen wurde in dieser Arbeit auch der zugehörige Pegel $L_{m E v}$ betrachtet. Für $L_{m E v}<100 \mathrm{dBSPL}$ wurden die besten Ergebnisse erzielt. Die Werte für $Q$ reichen von 0,47-0,79 (Median: $0,67)$ und streuen vergleichsweise wenig. Für $L_{m E v} \geq 100 \mathrm{~dB} S P L$ hingegen streuen die Ergebnisse deutlich mehr und für $Q$ ergaben sich Mediane zwischen 0,39 und 0,45. Dennoch konnten auch bei sehr hohen Werten für $L_{m E v} \geq 115 \mathrm{~dB}$ SPL noch vereinzelt relativ hohe Q-Werte erreicht werden. Statistische Analysen zeigten keine signifikanten Unterschiede zwischen den Gruppen im Bereich von 100 bis $>115$ dB SPL.

\section{Fazit für die Praxis}

- Für Hörverluste oberhalb $60 \mathrm{~dB}$ HL werden die Zielkurven für NAL-NL2 oder DSL v5.0 bei $65 \mathrm{~dB}$ SPL Eingangspegel zwar häufig erreicht, dennoch liegt das Sprachverstehen mit Hörgerät $\left(\mathrm{EV}_{65}(\mathrm{HG})\right)$ oft weit unter dem maximalen Einsilberverstehen (mEV).

- Der Wirkungsgrad Q der Hörgeräteversorgung, berechnet als Quotient aus $\mathrm{EV}_{65}(\mathrm{HG})$ und $\mathrm{mEV}$, liegt im Mittel bei 0,43 und gibt Auskunft darüber, inwieweit das Potenzial der Hörgeräteversorgung ausgeschöpft wurde.

- Die Hörgeräteeinstellungen, bei denen die Zielkurve für DSL v5.0 bei $65 \mathrm{~dB}$ SPL Eingangspegel erreicht wird, führen zu einem besseren $\mathrm{EV}_{65}(\mathrm{HG})$ und damit auch zu einem besseren $\mathrm{Q}$.

\section{Korrespondenzadresse}

\section{Max Engler, M.Sc.}

Audiologische Abteilung, Hals-NasenOhrenklinik, Kopf- und Halschirurgie, Universitätsklinikum Erlangen Waldstr. 1, 91054 Erlangen, Deutschland max.engler@uk-erlangen.de

Förderung. Diese Untersuchung wurde gefördert von der KIND Hörstiftung.

Funding. Open Access funding enabled and organized by Projekt DEAL.

\section{Effectiveness of hearing aid provision for severe hearing loss}

Background and objective: In practice, the unilateral monosyllabic speech recognition score with hearing aid $\left(\mathrm{WRS}_{65}(\mathrm{HA})\right)$ is often below the maximum word recognition score with headphones $\left(W R S_{\max }\right.$ ), in particular for subjects with severe hearing loss. The aim of this study was to evaluate the efficiency factor $Q$ of hearing aid provision, the ratio $W_{R S}{ }_{65}(\mathrm{HA}) / \mathrm{WRS}_{\max }$, in patients with severe to profound hearing loss.

Materials and methods: Data from real-ear measurements (REM), pure tone and speech audiogram, and speech recognition with and without hearing aid of 93 ears in 64 patients were examined. The patients visited the authors' hearing center for hearing aid evaluation in 2019. Deviations of the real-ear measured frequencydependent output level values from the prescription targets NAL-NL2 and DSL v5.0 were analyzed. Spearman correlation coefficients for the speech intelligibility index (SII) were calculated for the parameters $\mathrm{WRS}_{65}(\mathrm{HA})$ and $\mathrm{Q}$.

Results: In more than $67 \%$ of the hearing aid fittings, output level values matched the target curves of NAL-NL2 or DSL $v 5.0$ in the range of $\pm 5 \mathrm{~dB}$ for frequencies from 0.5 to $4 \mathrm{kHz}$ at $65 \mathrm{~dB}$ SPL. Nevertheless, WRS max $_{\text {was not }}$ nochieved with hearing aid at conversational speech levels of $65 \mathrm{~dB}$ SPL (mean deviations: $34.4 \%$ ). However, $W_{R S}{ }_{65}(\mathrm{HA})$ and $\mathrm{Q}$ were best when target values for DSL v5.0 were achieved at $65 \mathrm{~dB} S P L$, which is associated with a higher SII.

Conclusion: For patients with severe to profound hearing loss, the prescription targets of NAL-NL2 and DSL v5.0 do not provide sufficient amplification for $W_{\text {RS }}$ max to be achieved at a normal speech level of $65 \mathrm{~dB}$ SPL. It remains to be investigated whether alternative prescriptions with better audibility for input levels of 50 and $65 \mathrm{~dB}$ SPL might improve the effectiveness of hearing aid provision.

\section{Keywords}

Freiburg speech test $\cdot$ Maximum monosyllabic word recognition score $\cdot$ Speech audiometry $\cdot$ Realear measurements . Severe to profound hearing loss

\section{Einhaltung ethischer Richtlinien}

Interessenkonflikt. M. Engler, F. Digeser und U. Hoppe geben an, dass kein Interessenkonflikt besteht.

Alle beschriebenen Untersuchungen am Menschen oder an menschlichem Gewebe wurden mit Zustimmung der zuständigen Ethikkommission, im Einklang mit nationalem Recht sowie gemäß der Deklaration von Helsinki von 1975 (in der aktuellen, überarbeiteten Fassung) durchgeführt. Von allen beteiligten Patienten liegt eine Einverständniserklärung vor. Die Studie wurde von der Ethik-Kommission der FriedrichAlexander-Universität Erlangen positiv beschieden (Nr. 162_17 Bc).

Open Access. Dieser Artikel wird unter der Creative Commons Namensnennung 4.0 International Lizenz veröffentlicht, welche die Nutzung, Vervielfältigung, Bearbeitung, Verbreitung und Wiedergabe in jeglichem Medium und Format erlaubt, sofern Sie den/die ursprünglichen Autor(en) und die Quelle ordnungsgemäß nennen, einen Link zur Creative Commons Lizenz beifügen und angeben, ob Änderungen vorgenommen wurden.

Die in diesem Artikel enthaltenen Bilder und sonstiges Drittmaterial unterliegen ebenfalls der genannten Creative Commons Lizenz, sofern sich aus der Abbildungslegende nichts anderes ergibt. Sofern das betreffende Material nicht unter der genannten Creative Commons Lizenz steht und die betreffende Handlung nicht nach gesetzlichen Vorschriften erlaubt ist, ist für die oben aufgeführten Weiterverwendungen des Ma- terials die Einwilligung des jeweiligen Rechteinhabers einzuholen.

Weitere Details zur Lizenz entnehmen Sie bitte der Lizenzinformation auf http://creativecommons.org/ licenses/by/4.0/deed.de.

\section{Literatur}

1. ANSI (1997)R2017S3.5 Methodsfor the Calculation of the Speech Intelligibility Index. Acoustical Society of America, New York

2. ANSI (2004) S3.6 American national standard specification for audiometers. Acoustical Society of America, New York

3. Cornelisse LE, Seewald RC, Jamieson DG (1995) The input/output formula: a theoretical approach to the fitting of personal amplification devices. JAcoust Soc Am 97(3):1854-1864

4. Digeser FM, Engler M, Hoppe U (2020) Comparison of bimodal benefit for the use of DSL v5.0 and NAL-NL2 in cochlear implant listeners. Int J Audiol 59(5):383-391

5. Dörfler C, Hocke T, Hast A, Hoppe U (2020) Sprachverstehen mit Hörgeräten für 10 Standardaudiogramme. HNO 68:40-47

6. Gemeinsamer Bundesausschuss (2020) Hilfsmittel-Richtlinie über die Verordnung von Hilfsmitteln in der vertragsärztlichen Versorgung (HilfsmittelRichtlinie / HilfsM-RL) in der Neufassung vom 17.09.2020. BAnz AT 30.09.2020 B2. Gemeinsamer Bundesausschuss, Berlin 


\section{In eigener Sache}

7. Hahlbrock KH (1953) Über Sprachaudiometrie und neue Wörterteste. Arch Ohren Nasen Kehlkopfheilkd 162(5):394-431

8. Holube I, Fredelake S, Vlaming M, Kollmeier B (2010) Development and analysis of an international speech test signal (ISTS). Int J Audiol 49(12):891-903

9. Hoppe U, Hast A, Hocke T (2014) Sprachverstehen mit Hörgeräten in Abhängigkeit vom Tongehör. HNO 62(6):443-448

10. Hoppe U (2016) Hörgeräteerfolgskontrolle mit dem Freiburger Einsilbertest. HNO64(8):589-594

11. https://partners.natus.com/asset/resource/ file/otometrics/asset/2021-03/7-26-1200-DE_ Rev01_WEB.pdf.Zugegriffen:4.Nov.2021

12. Keidser G, Dillon H, Flax M, Ching T, Brewer S (2011) The NAL-NL2 prescription procedure. Audiol Res 1:88-90

13. Kießling J (2018) Konventionelle Hörsysteme: Hörgeräte.In:Kießling J, Kollmeier B, BaumannU(Hrsg) Versorgung mit Hörgeräten und Hörimplantaten, 3. Aufl. Thieme, Stuttgart

14. Kronlachner M, Baumann U, Stöver T, Weißgerber T (2018) Untersuchung der Qualität der Hörgeräteversorgung bei Senioren unter Berücksichtigung kognitiver Einflussfaktoren. Laryngorhinootologie 97(12):852-859

15. Löhler J, Walther LE, Hansen F, Kapp P, Meerpohl J, Wollenberg B, Schmucker C (2019) The prevalence of hearing loss and use of hearing aids among adults in Germany: a systematic review. Eur Arch Otorhinolaryngol 276(4):945-956

16. Moore BC, Glasberg BR (2004) A revised model of loudness perception applied to cochlear hearing loss. Hear Res 188(1-2):70-88

17. Müller A, Hocke T, Hoppe U, Mir-Salim P (2016) Der Einfluss des Alters bei der Evaluierung des funktionellen Hörgerätenutzens mittels Sprachaudiometrie. HNO 64(3):143-148

18. Mueller HG (2014) 20Q: Real-ear probe-microphone measures-30 years of progress? Audiology Online, Article 12410. https://www. audiologyonline.com/articles/20q-probe-micmeasures-12410.Zugegriffen:22.0kt. 2021

19. Schorn K (2004) Hörgeräteüberprüfung in der Praxis. HNO52(10):875-885

20. Schorn K (2006) Die Aufgaben des Hals-NasenOhren-Arztes bei der Hörgeräteversorgung. HNO 54(3):233-251

21. Scollie S, Seewald R, Cornelisse L, Moodie S, Bagatto M, Laurnagaray D, Beaulac S, Pumford J (2005) The desired sensation level multistage input/ output algorithm. Trends Amplif 9(4):159-197

22. von Gablenz P, Holube I (2015) Prevalence of hearing impairment in northwestern Germany. Results of an epidemiological study on hearing status (HÖRSTAT). HNO63(3):195-214

23. von Gablenz P, Hoffmann E, Holube I (2017) Prevalence of hearing loss in Northern and Southern Germany. HNO 65(2):130-135

24. Winkler A, Holube I (2016) Test-Retest-Reliabilität des Freiburger Einsilbertests. HNO64(8):564-571

25. World Health Organization (Hrsg) (2021) World report on hearing. World Health Organization Geneva (Licence: CCBY-NC-SA 3.0 IGO)

Die Zeitschrift HNO bietet Ihnen jeden Monat umfassende und aktuelle Beiträge zu interessanten Themenschwerpunkten aus allen Bereichen der Hals-, Nasen-, Ohrenheilkunde, Kopf- und Halschirurgie.

$\begin{array}{ll}\text { - 01/2022 } & \text { Varia } \\ \text { - 02/2022 } & \text { Varia } \\ \text { - 03/2022 } & \text { Varia } \\ \text { - 04/2022 } & \text { Post-ASCO/ESMO 2021 } \\ \text { - 05/2022 } & \text { Kongressheft } \\ \text { - 06/2022 } & \text { Varia } \\ \text { - 07/2022 } & \text { Varia } \\ \text { - 08/2022 } & \text { Speicheldrüsenkarzinom }\end{array}$

Alle Inhalte von $H N O$ finden Sie unter

www.springermedizin.de/hno-zeitschrift.

SpringerMedizin.de bietet Ihnen Zugang zu allen elektronisch verfügbaren Ausgaben Ihrer Zeitschrift - unabhängig vom Beginn Ihres Abonnements.

Möchten Sie ein bereits erschienenes Heft nachbestellen? Einzelne Ausgaben können Sie direkt bei unserem Kundenservice zum Preis von je EUR 46,- zzgl. Versandkosten beziehen.

\section{So erreichen Sie unseren Kundenservice:}

Springer Customer Service Center GmbH

Kundenservice Zeitschriften

Tiergartenstr. 15, 69126 Heidelberg

Tel.: +49 $6221345-4303$

Fax: $+496221345-4229$

E-Mail: leserservice@springer.com 